\section{Topical cyclosporine A for the dry eye findings of thyroid orbitopathy patients}

\begin{abstract}
Purpose To determine the beneficial effect of topically administered Cyclosporine A (CsA) for the dry eye findings of thyroid orbitopathy patients.

Patients and methods This prospective pilot study included 73 eyes of 42 patients with thyroid orbitopathy who had documented dry eye findings. Patients were randomly assigned into two groups: in group 1 (48 eyes), patients received topical artificial tear-drop treatment. In group 2 (25 eyes), patients received topical CsA and artificial tear-drop treatment. During a mean follow-up of 6 months, change in Schirmer's test with aneasthesia, tear break-up-time (BUT) and impression cytology results were analyzed and were compared between groups. Results The two groups were age $(P=0.449)$ and gender $(P=0.942)$ matched.
\end{abstract} The Schirmer's test $(P=0.441)$, tear BUT $(P=0.718)$ and impression score $(P=0.103)$ were also similar before the treatment in both groups. In group 1, all three parameters improved significantly with treatment $(P<0.001$ for all). In group 2, Schirmer's test $(P=0.001)$ and tear BUT $(P<0.001)$ improved, but the impression score $(P=0.175)$ did not change significantly after treatment. The percentage of patients with improved tear BUT $(P=0.04)$ and improved impression score $(P<0.001)$ were higher in group 1 . At the end of follow-up, group 1 patients had better Schirmer's test $(P=0.004)$, tear BUT $(P=0.021)$ and impression scores $(P<0.001)$, than group 2 patients.

Conclusions The combined CsA use with artificial tear drops is not more advantageous than the use of artificial tear drops alone, for the dry eye findings of thyroid orbitopathy patients.

Eye (2010) 24, 1044-1050; doi:10.1038/eye.2009.246;

published online 16 October 2009
UE Altiparmak¹, DE Acar ${ }^{1}$, PA Ozer ${ }^{1}$, SD Emec ${ }^{1}$, R Kasim¹, H Ustun² and S Duman ${ }^{1}$

Keywords: thyroid orbitopathy; dry eye; cyclosporine A; artifical tears

\section{Introduction}

Thyroid orbitopathy (TO) is an organ-specific autoimmune disorder and is characterized by inflammation, oedema and fibrosis at the orbital tissue. Extraocular muscles, orbital tissues and lacrimal glands are target tissues for natural killer (NK) cells, T- and B- lymphocytes which become sensitive to the thyroid tissue. Lymphocyte and plasma-cell infiltration, glycosaminoglycan accumulation, oedema followed by fibrosis are histopathological findings seen at these target tissues. ${ }^{1}$ The signs and symptoms of TO are the results of increased volume of the orbital contents by oedema, hydrophilic glycosaminoglycans and adipose tissue. ${ }^{1}$ Yet, the exact pathophysiology of Graves' disease still remains enigmatic. Therefore, some signs and symptoms of the disease are not fully explained to date.

Dry-eye symptoms are commonly seen in patients with $\mathrm{TO}^{2-4}$ The most likely mechanism is the increased evaporation of the tear film in TO patients, resulting in evaporative dry eye (EDE).$^{2-4}$ This is the result of increase in volume of the orbital contents that push the globe anteriorly and increase the palpebral fissure width. Proptosis (and lid retraction, if present) secondarily causes evaporative loss of the tear film and increases the tear film osmolarity.

Recent evidence demonstrated additional possible mechanisms that may contribute to the pathogenesis of dry-eye symptoms. According to this theory, EDE may take the features of aqueous-deficient dry eye (ADDE) as the disease evolves and vice versa. ${ }^{5}$ As a consequence, early and late forms may require different diagnostic criteria and respond to different therapeutic regimes. ${ }^{5}$ 
Cyclosporine A (CsA) is an immunomodulator drug that inhibits interleukin (IL)-2 and prevents the activation of cytotoxic T-cell response. It has been traditionally used to prevent the rejection of organ transplantation and for the treatment of autoimmune disorders. In recent years, it has been used for the treatment of dry-eye symptoms in its advanced stages. ${ }^{6-11}$

In this pilot study, we combined the use of CsA with artificial tear drops in a group of TO patients with documented dry-eye symptoms and compared its use with artificial tear drops alone, in an attempt to reduce dry-eye symptoms more effectively.

\section{Materials and methods}

The study was approved by the local ethics committee of Ministry of Health, Ankara Research and Training Hospital (31 May 2005, Number. 150 821) and patient recruitment began. The authors declared that they have no conflict of interest. A total of 90 eyes of 45 patients with TO were prospectively evaluated for this study between May 2005 and December 2007. The diagnosis of TO was made, when two of the following three signs of the disease were present: ${ }^{12}$ (1) Concurrent or recently treated immune-related thyroid dysfunction: Graves hyperthyroidism, Hashimoto's thyroiditis, and the presence of circulating thyroid antibodies without a coexisting dysthyroid state; (2) typical orbital signs (one or more of the following): unilateral or bilateral eyelid retraction with typical temporal flare (with/or without lagophthalmos), bilateral proptosis (as evidenced by comparison with patient's old photos), restrictive strabismus in a typical pattern compressive optic neuropathy, fluctuating eyelid oedema/erythema, chemosis/caruncular oedema; and (3) radiographic evidence of TO including unilateral/bilateral fusiform enlargement of one or more of the following: medial rectus muscle, inferior rectus muscle, superior rectuslevator complex. In all patients, assessment of activity of TO was carried out using Clinical Activity Score (CAS) based on the classification system proposed by Mourits et al. ${ }^{13}$

All patients underwent a thorough ophthalmic examination, including tear function analysis using tear break-up-time (BUT) and Schirmer's test with aneasthesia, as well as impression cytology by the same ophthalmologist (PAO). Tear BUT test was determined by the application of a strip of moistened fluorescein strip in the lower eyelid fornix. The patient was asked to blink three times and then look straight forward, without blinking. The tear film was observed under the broad beam of the slit-lamp microscope with a blue filter. The time lapse between the last blink and appearance of the first randomly distributed dry spot in the cornea was taken as the tear BUT. Tear BUT was measured three times, and the mean value of the measurements was calculated. Dry spots appearing in $10 \mathrm{~s}$ or less were considered as disturbed tear stability.

Schirmer's test of the basic secretion variety was carried out as follows: following topical anaesthesia using proparacaine $\% 0.5$ eye drops, residual fluid was blotted out of the inferior fornix lightly. A thin filterpaper strip ( $5 \mathrm{~mm}$ wide, $35 \mathrm{~mm}$ long) was placed at the junction of the middle and lateral thirds of the lower eyelids to minimize irritation to the cornea during the test. Test was carried out with the eyes closed, to limit the effect of blinking. Patients with a BUT of $<10$ s and Schirmer's test of $<10 \mathrm{~mm}$ were considered as dry eye and were included in the study.

After topical anaesthesia with proparacaine $\% 0.5$ eye drops conjunctival and/or corneal cytology impressions were obtained by using a single packed, sterile Biopore membrane (Millicell-CM 0.4 $\mu \mathrm{m}$ PICM 012550, Millipore, Bedford, MA, USA). To increase the number of cells that were harvested, the ocular surface was slightly dried with a cotton swab or by keeping the eye open for some time before impression cytology was carried out. Using a pair of fine forceps, cellulose acetate strips were applied to the bulbar conjunctiva. The strips were then gently peeled off lifting the edge with fine forceps. The samples were fixed in $95 \%$ ethanol, stained with periodic acid Schiff (PAS), and examined in a Zeiss Axioplan light microscope, and graded by a masked observer (HU). Impression cytology was evaluated by looking at the morphological changes on epithelial cells, anisocytosis, nucleus : cytoplasm ratio, presence of structural changes of the nuclear chromatin (snake-like chromatin) and the change in goblet cell density. In accordance with this method, patients were graded on a scale from 0 to 3, as described by Nelson ${ }^{14}$ (Table 1).

Patients with a CAS of 4 and above (presence of clinically active TO) were left out of the study, to avoid interference with TO symptoms. For all patients, presence of conjunctival tumours, corneal diseases that may influence conjunctival vascularization, glaucoma, a history of previous ocular surgery, as well as persistent meibomian gland dysfunction were other criteria for exclusion. Patients enrolled were not using any topical medication before the study. Eyes rather than persons were used as the unit for statistical analysis.

Patients qualified for the study were randomly assigned into two treatment groups (by UEA). To the first group only artificial tear drops four times a day (Refresh Tears, Allergan, Waco, TX, USA), to the second group artificial tear drops (Refresh Tears, Allergan, Waco, TX, USA) and topical CsA 0.05\% (Restasis, Allergan, Waco, TX, USA) twice a day were started. Patients were seen 6 months later by a masked observer (DEA) to the 
Table 1 Nelson staging system for impression cytology of the conjunctiva ${ }^{15}$

\begin{tabular}{llll}
\hline Stage & Epithelial cells & $\begin{array}{l}\text { Nucleus:cytoplasm } \\
\text { ratio }\end{array}$ & $\begin{array}{l}\text { Goblet cells } \\
0\end{array}$ \\
\hline & $\begin{array}{l}\text { Small and round. The cytoplasm is coloured } \\
\text { and eosinophilic, and the nucleus is big and } \\
\text { basophilic. }\end{array}$ & $1: 2$ & $\begin{array}{l}\text { Excessive in number, dense, filled, ovale and have PAS } \\
\text { positive cytoplasm. }\end{array}$ \\
$\begin{array}{l}\text { Bigger and polygonal. The cytoplasm is } \\
\text { eosinophilic and the nucleus starts to grow. }\end{array}$ & $1: 3$ & $\begin{array}{l}\text { Although decreased in number, their sizes are the } \\
\text { same and they are filled, ovale, and have PAS positive } \\
\text { cytoplasm. } \\
\text { Decreased significantly in number, became smaller } \\
\text { and started to disappear. More lightly coloured PAS } \\
\text { positive cytoplasm is found. } \\
\text { Very low counts or completely disappeared. }\end{array}$ \\
$\begin{array}{l}\text { Much bigger and polygonal. The cytoplasm is } 1: 4-1: 5 \\
\text { coloured variably and multinucleus is found } \\
\text { rarely. }\end{array}$ & $\begin{array}{l}\text { Biggest and polygonal. Their colours are bright } \\
\text { and show folding. The cytoplasm is basophilic. } \\
\text { The nucleus is small, picnotic, and is not found } \\
\text { in most cells. }\end{array}$ \\
\hline
\end{tabular}

Abbreviation: PAS, periodic acid Schiff

treatments and the same measurements (including impression cytology specimens) were obtained. Improvement in the test measures was the primary outcome measure.

For analysis of data, Statistical Package for Social Sciences, version 13.0 (SPSS, Chicago, IL, USA) software program was used. Continuous variables were expressed as mean $\pm \mathrm{SD}$ and categorical variables were expressed as percentages. Mann-Whitney $U$-test, Wilcoxon's signedrank test and Pearson's $\chi^{2}$ tests were used for analysis and $P$-values $<0.05$ were considered statistically significant.

\section{Results}

The first group initially consisted of 50 eyes of 25 patients but 1 patient was lost to follow-up. The second group initially consisted of 40 eyes of 20 patients, 3 patients (6 eyes) were lost to follow-up and topical CsA was discontinued in another 9 eyes because of intense burning sensation. Finally, 48 eyes remained in the first cohort and 25 eyes remained in the second cohort.

The mean age of the study group was $41.6 \pm 9.8$ years. The mean BUT at onset increased significantly (from $6.26 \pm 1.82$ to $12.22 \pm 3.74$ ), as well as mean Schirmer's test with anaesthesia (from $3.59 \pm 1.9$ to $9.34 \pm 5.57$ ) and impression score (from $1.85 \pm 0.68$ to $1.42 \pm 0.82$ ) until the end of the study $(P<0.001$ for all three analyses, Table 2$)$.

When the two study cohorts were analyzed, they were age $(P=0.929)$ and gender-matched $(P=0.942)$ and the measurement of BUT $(P=0.718)$, Schirmer's test with anaesthesia $(P=0.441)$ and impression score at onset $(P=0.103)$ were similar (Table 3$)$.

In group 1, mean Schirmer's test with anaesthesia at onset was $3.73 \pm 1.85 \mathrm{~mm}$ and improved to $10.73 \pm 5.71$ at the end of follow-up $(P<0.001)$. In the same group,
Table 2 General properties of the study group and the results of study parameters

\begin{tabular}{lcc}
\hline Study parameter & Mean $\pm S D($ range $)$ & P-value $^{\text {a }}$ \\
\hline Schirmer's test at onset (mm) & $3.59 \pm 1.9(1.0-9.0)$ & $<0.001$ \\
Schirmer's test final (mm) & $9.34 \pm 5.57(1.0-20.0)$ & \\
BUT at onset (s) & $6.26 \pm 1.82(3.0-10.0)$ & $<0.001$ \\
BUT final (s) & $12.22 \pm 3.74(5.0-20.0)$ & \\
Impression score at onset & $1.85 \pm 0.68(0.0-3.0)$ & $<0.001$ \\
Impression score final & $1.42 \pm 0.82(0.0-3.0)$ & \\
\hline
\end{tabular}

Abbreviations: BUT, break-up time; $\mathrm{mm}$, milimeters; s, seconds; SD, standard deviation.

${ }^{a}$ Wilcoxon's signed-rank test.

BUT at onset $(6.21 \pm 1.73)$ also improved (12.9 \pm 3.96$)$ significantly at the end of follow-up period $(P<0.001)$. Similarly, the impression score of group 1 improved from $1.94 \pm 0.6$ to $1.17 \pm 0.72$, within the same time period $(P<0.001)$.

In group 2, mean Schirmer's test with anaesthesia at onset was $3.32 \pm 1.99 \mathrm{~mm}$ and improved to $6.68 \pm 4.24 \mathrm{~mm}$ at the end of follow-up $(P=0.001)$. In the same group BUT at onset was $6.36 \pm 2.02$ also improved to $10.92 \pm 2.94$ at the end of follow-up $(P<0.001)$. In contrast, the impression score at onset $(1.68 \pm 0.8)$ did not change at the end of follow-up period (1.92 $\pm 0.76, P=0.175)$.

In group 1, 47 eyes (97.9\%) had improved BUT score, compared with 22 eyes $(88.0 \%)$ in group 2 (Figure 1, $P=0.04)$. Schirmer's test score improved in 43 eyes $(89.6 \%)$ in group 1 and 22 eyes $(88.0 \%)$ in group 2 (Figure 2, $P=0.893$ ). Finally, impression scores improved in 35 eyes $(72.9 \%)$ in group 1 and 5 eyes (20.0\%) in group 2 (Figure 3, $P<0.001$ ).

At the end of the follow-up period, the first group, where patients used only artificial tears had better Schirmer's test, BUT and impression scores $(P=0.004$, 0.021 and $<0.001$, respectively, Table 3 ). 
Table 3 Comparison of two study sub-groups, based on treatment

\begin{tabular}{lccr}
\hline Study parameter (mean $\pm S D$, range) & Group 1 $(\mathrm{n}=48)$ & Group 2 $(\mathrm{n}=25)$ & P-value \\
\hline Age & $40.9 \pm 8.8(28-66)$ & $41 \pm 1.1(23-65)$ & $0.449^{\mathrm{a}}$ \\
Gender (male/female) & $8 / 40$ & $4 / 21$ & $0.942^{\mathrm{b}}$ \\
Schirmer's test at onset (mm) & $3.73 \pm 1.85(1-8)$ & $3.32 \pm 1.99(1-9)$ & $0.441^{\mathrm{a}}$ \\
Schirmer's test final (mm) & $10.73 \pm 5.71(1.0-20.0)$ & $6.68 \pm 4.24(1.0-20.0)$ & $0.004^{\mathrm{a}}$ \\
BUT at onset (s) & $6.21 \pm 1.73(4.0-10.0)$ & $6.36 \pm 2.02(3.0-10)$ & $0.718^{\mathrm{a}}$ \\
BUT final (s) & $12.9 \pm 3.96(5.0-20.0)$ & $10.92 \pm 2.94(5.0-15.0)$ & $0.021^{\mathrm{a}}$ \\
Impression score at onset & $1.94 \pm 0.6(0-3)$ & $1.68 \pm 0.8(0-3)$ & $0.103^{\mathrm{a}}$ \\
Impression score final & $1.17 \pm 0.72(0-3)$ & $1.92 \pm 0.76(0-3)$ & $<0.001^{\mathrm{a}}$ \\
Patients with improved BUT (\%) & $47(97.9 \%)$ & $22(88.0 \%)$ & $0.04^{\mathrm{b}}$ \\
Patients with improved Schirmer's test $(\%)$ & $43(89.6 \%)$ & $22(88.0 \%)$ & $0.893^{\mathrm{b}}$ \\
Patients with improved impression Score (\%) & $35(72.9 \%)$ & $5(20.0 \%)$ & $<0.001^{\mathrm{b}}$ \\
\hline
\end{tabular}

Abbreviations: BUT, break-up time; mm, milimeters; s, seconds; SD, standard deviation.

Group 1 received artifical tear drops, group 2 received topical Cyclosporine A and artifical tear drops.

a'Mann-Whitney $U$-test.

'Pearson's $\chi^{2}$ test.

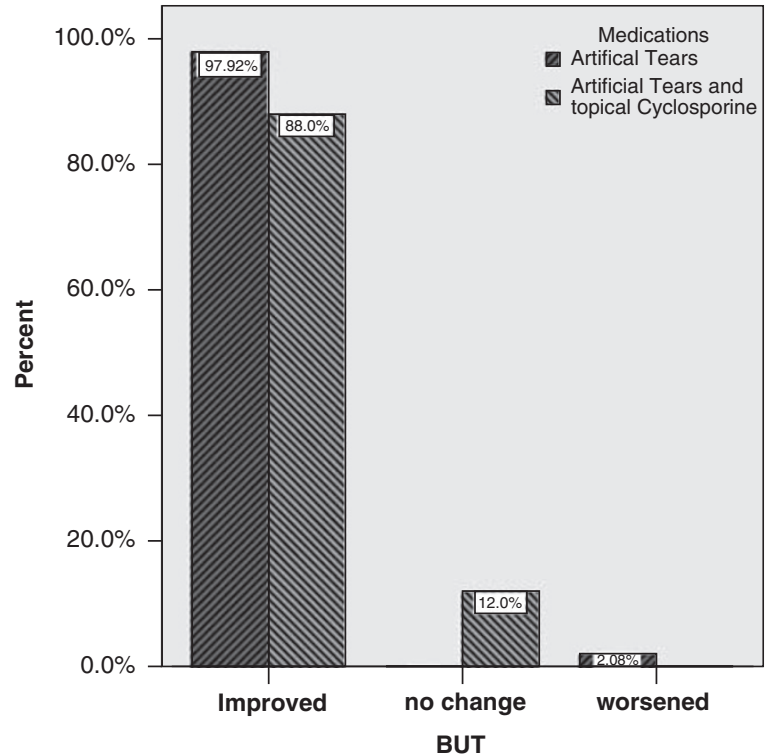

Figure 1 Change in tear break-up-time (BUT) with the medications is seen $\left(P=0.04\right.$, Pearson's $\chi^{2}$ test $)$.

\section{Discussion}

In this study, clinically inactive TO patients with dry eye were randomly enrolled into two different treatment groups, as artificial tear drops alone or artificial tear drops combined with topical CsA. Patients with underlying meibomian disease were excluded, as well as other ocular diseases that may affect ocular surface. To avoid selection bias, only patients with moderate-tosevere dry-eye symptoms were included, namely, those with both decreased basic tear secretion and disturbed tear stability. In addition, conjunctival impression cytology, which is a safe, relatively simple, and painless method of obtaining specimens from the conjunctival

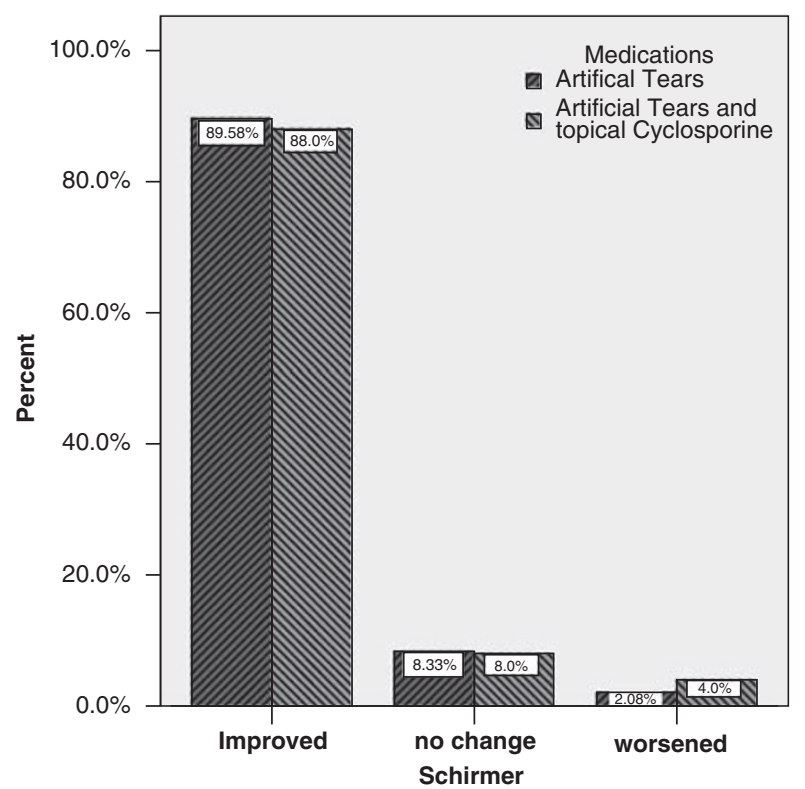

Figure 2 Change in tear Schirmer's test with the medications is seen $\left(P=0.893\right.$, Pearson's $\chi^{2}$ test).

surface was also carried out. ${ }^{14}$ Most treatment parameters improved significantly in both groups, except the impression score of patients using artificial tear drops and topical CsA. Similarly, more patients using artificial tear drops alone had improved impression and BUT score than patients using topical CsA in addition to artificial tear drops. Though both treatment groups had similar test scores at the beginning of the study, patients treated with artificial tear drops alone had better test scores at the end of the follow-up period.

Evaporative loss is the proposed mechanism in the pathogenesis of dry-eye symptoms in TO patients. EDE occurs after increased proptosis secondary to increased 


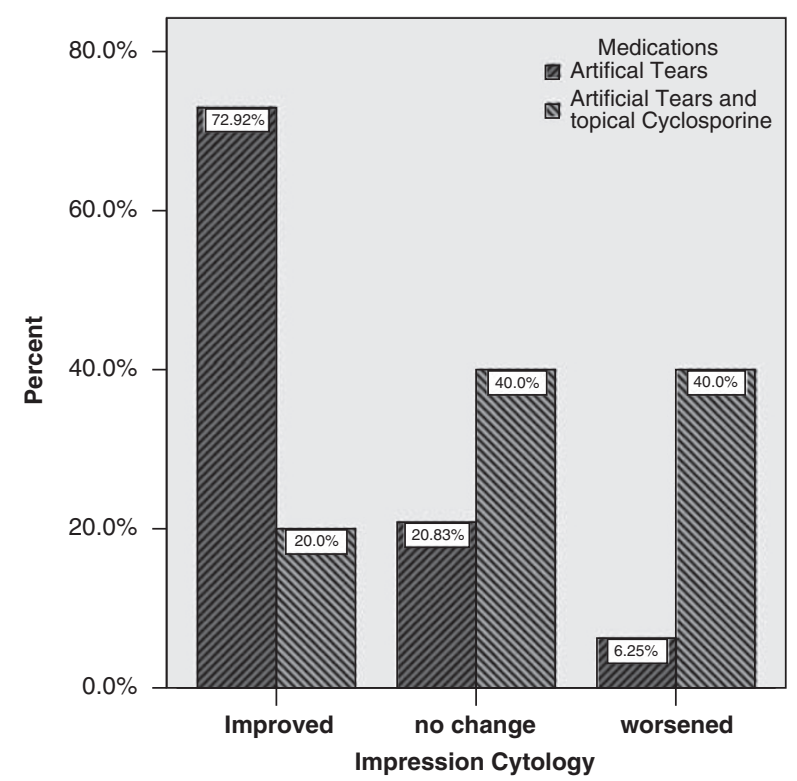

Figure 3 Change in impression cytology scores with the medications is seen $\left(P<0.001\right.$, Pearson's $\chi^{2}$ test $)$.

orbital contents. Correlation with increased palpebral fissure height and increased tear film osmolarity ${ }^{4}$ and decreased tear BUT $^{2}$ has been demonstrated in TO patients. The tear content is also changed in TO patients; Khalil and friends showed that the IgA: lysozyme ratio increased approximately $33 \%$ at thyroid ophthalmopathy patients. ${ }^{15}$ Nevertheless, as seen in our patients at the beginning of the study, all patients had decreased Schirmer's test score, similar to ADDE, suggesting aqueous deficiency. In the work of Bron $\mathrm{AJ} e t a l^{5}$ this phenotype of EDE is explained as a result of reduced corneal sensation and loss of the reflex compensatory increase in lacrimal secretion. This progressive reduction in lacrimal secretion results in a parallel, progressive, further rise in tear osmolarity, even before lacrimal secretion has fallen below normal levels. This could be described as a relative aqueous tear deficiency. ${ }^{5}$ A further decrease in lacrimal secretion to levels encountered in ADDE using standard diagnostic criteria could be described as an absolute deficiency. In this way, it is conceivable that EDE, at the stage of reflex decompensation, would evolve into a hybrid state in which EDE would be combined with a functional ADDE, which would at first be relative and then absolute.

In a more severe form of EDE, decreased secretion from lacrimal gland due to corneal damage and loss of reflex sensory drive causes a reduction in secretion. ${ }^{5}$ This is not caused by lacrimal gland obstruction or dysfunction, as one may expect to find in TO. ${ }^{16-19}$ Lacrimal proteins of acinar and extra-acinar origin, such as secretory IgA could be demonstrated at normal levels in such patients. ${ }^{5,20}$ Unfortunately, the analysis of lacrimal content could not be carried out in this study because of technical reasons. Nevertheless, therapeutic opportunities such as for the use of lacrimal secretagogues, ${ }^{21-23}$ or treatment directed towards the restoration of corneal sensory function and sensory drive would be beneficial.

Topical use of CsA in dry-eye syndrome was first reported by Laibovitz, et al. ${ }^{6}$ CsA effectively reduced lymphocyte infiltration and apoptosis of lacrimal gland and conjunctiva, and stimulated the apoptosis of lymphocytes in animal models with dry eye. ${ }^{24} \mathrm{Cs}$ A also significantly reduced IL-6 levels ${ }^{25}$ and HLA-DR and CD11A expression in conjunctival samples and increased goblet cell counts ${ }^{26}$ in dry eye patients. Topically instilled CsA increased conjunctival mucin storages in about 4 weeks duration, in canine keratoconjunctivitis sicca model. ${ }^{27}$ Stevenson et $a l^{8}$ demonstrated a significant reduction of Rose Bengal dye uptake in the conjunctiva and reduction of ocular surface disease index (OSDI) after the use of CsA in patients with dry eye. Moon $e t a l^{10}$ showed that CsA treatment in dry-eye syndrome provides stabilization of tear film layer and increases production of aqueous layer of the tear film layer. As a summary, the beneficial effects of CsA in dry eye patients are the result of reduced T-cell activation and conjunctival and lacrimal gland inflammation. ${ }^{11}$

Kunert et $a l,{ }^{26}$ showed increase in conjunctival goblet cells density and enhanced epithelial regeneration by topical cyclosporine A treatment in about six month. Also Strong et $\mathrm{l}^{28}$ demonstrated decreased conjunctival epithelial cell apoptosis by topical CsA and prevention of goblet cell loss.

Several reports have evaluated the use of CsA administration in TO. Among the few studies that were randomized and controlled, CsA was found to have a lower effect than prednisone as a single-agent treatment but a stronger effect than prednisone or CsA alone, when used in combination. ${ }^{29,30}$ To the best of our knowledge, our study is the first about the effects of topical CsA on the dry eye findings of TO patients.

One weakness of our study is the low number of patients and unequal distribution into two groups because of patient discontinuation to medication or losses to follow-up, which influenced the second group patients more significantly. Hence, we consider our cohort as a pilot study, to study the effectiveness of CsA in dry-eye symptoms of TO patients.

The results of this pilot study suggest that topical CsA does not provide additional benefit over the use of artificial tear drops alone, for the treatment of dry eye findings of TO patients. We are unsure about the underlying mechanism of this result. Yet, it can be 
speculated that the functional ADDE, which develops as EDE progresses, limits the effectiveness of topical CsA in TO patients. Lacrimal secretagogues in TO patients with dry-eye symptoms could be superior to topical CsA to improve tear function. We believe further studies that focus in this area would provide explanatory and supplementary data on this subject.

\section{Summary}

\section{What was known before}

- Cyclosporine $\mathrm{A}$ is an effective treatment option in patients with dry eye symptoms.

\section{What this study adds}

- Cyclosporine A does not provide additional effect over artifical tear drops alone, in thyroid orbitopathy patients with dry-eye symptoms.

\section{Conflict of interest}

The authors declare no conflict of interest.

\section{References}

1 Ludgate M, Baker G. Unlocking the immunological mechanisms of orbital inflammation in thyroid eye disease. Clin Exp Immunol 2002; 127: 193-198.

2 Gilbard JP, Farris RL. Ocular surface drying and tear film osmolarity in thyroid eye disease. Acta Ophthalmol 1983; 61 108-116.

3 Khurana AK, Sunder S, Ahluwalia BK, Malhotra KC. Tear film profile in Graves ophthalmopahy. Acta Ophthalmol 1992; 70: 346-349.

4 Brasil MV, Brasil OF, Vieira RP, Vaisman M, Amaral Filho OM. Tear film analysis and its relation with palpebral fissure height and exophthalmos in Graves' ophthalmopathy. Arq Bras Oftalmol 2005; 68(5): 615-618.

5 Bron AJ, Yokoi N, Gafney E, Tiffany JM. Predicted phenotypes of dry eye: proposed consequences of its natural history. Ocul Surf 2009; 7(2): 78-92.

6 Laibovitz RA, Solch S, Andriano K, O'Connell M, Silverman $\mathrm{MH}$. Pilot trial of cyclosporine 1\% ophthalmic ointment in the treatment of keratoconjunctivitis sicca. Cornea 1993; 12(4): 315-323.

7 Sall K, Stevenson OD, Mundorf TK, Reis BL. Two multicenter, randomized studies of the efficacy and safety of cyclosporineophthalmic emulsion in moderate to severe dry eye disease. CsA Phase 3 Study Group. Ophtalmology 2000; 107(7): 631-639.

8 Stevenson D, Tauber J, Reis BL. Efficacy and safety of cyclosporin A ophthalmic emulsion in the treatment of moderate-to-severe dry eye disease: a dose-ranging, randomized trial. The Cyclosporin A Phase 2 Study Group. Ophtalmology 2000; 107(5): 967-974.

9 Perry HD, Donnenfeld ED. Topical \%0.05 Cyclosporin in the treatment of dry eye. Expert Opin Pharmacother 2004; 5(10): 2099-2107.
10 Moon JW, Lee HJ, Shin KC, Wee WR, Lee JH, Kim MK. Short term effects of topical cyclosporine and viscoelastic on the ocular surfaces in patients with dry eye. Korean $J$ of Ophthalmol 2007; 21(4): 189-194.

11 Stephen C. Pflugfelder: antiinflammatory therapy for dry eye. Am J Ophthalmol 2004; 137: 337-342.

12 Holds JB, Chang WJ, Dailey RA, Foster JA, Kazim M, McCulley TJ et al. Infectious and Inflammatory Disorders. In: Liesegang TJ, Skuta GL, Cantor LB (eds). Basic and Clinical Science Course Section 7: Orbit, Eyelids, and Lacrimal System. American Academy of Ophthalmology: San Francisco, CA, USA, 2007-2008; 39-58.

13 Mourits M, Prummel MF, Wiersinga WM, Koornneef L. Clinical Activity Score as a guide in the management of patients with Graves' ophthalmopathy. Clinical Endocrinol 1997; 47: 9-14.

14 Nelson JD. Impression cytology. Cornea 1988; 7(1): 71-81.

15 Khalil HA, De Keizer RJ, Bodelier VM, Kijlstra A. Secretory IgA and lysozyme in tears of patients with Graves ophthalmopathy. Doc Ophthalmol 1989; 72: 329-334.

16 Chang TC, Huang KM, Chang TJ, Lin SL. Correlation of orbital computed tomography and antibodies in patients with hyperthyroid Graves' disease. Clin Endocrinol (Oxf) 1990; 32(5): 551-558.

17 Moncayo R, Baldissera I, Decristoforo C, Kendler D, Donnemiller E. Evaluation of immunological mechanisms mediating thyroid-associated ophthalmopathy by radionuclid imaging using the somatostatin analogue 111In-octreotid. Thyroid 1997; 7(1): 21-29.

18 Krenning EP, Kwekkeboom DJ, Bakker WH, Breeman WAP, Kooij PPM, Oei HY et al. Somatostatin receptor scintigraphy with ( ${ }^{111}$ In-DTPA-D-Phe1)-and (1231-Tyr3)-Octreotide:the Rotterdam experience with more than 100 patients. Eur J Nucl Med 1993; 20: 716-731.

19 Kahaly G, Diaz M, Hahn K, Beyer J, Bockish A. Indium-111pentreotide scintigraphy in Graves' ophthalmopathy. J Nucl Med 1995; 36: 550-554.

20 Sack RA, Beaton A, Sathe S, Morris C, Willcox M, Bogart B. Towards a closed eye model of the pre-ocular tear layer. Prog Retin Eye Res 2000; 19: 649-668.

21 Dartt DA, Matkin C, Gray K. Comparison of proteins in lacrimal gland fluid secreted in response to different stimuli. Invest Ophthalmol Vis Sci 1988; 29: 991-995.

22 Gilbard JP, Rossi SR, Heyda KG, Dartt DA. Stimulation of tear secretion and treatment of dry-eye disease with 3-isobutyl-1-methylxanthine. Arch Ophthalmol 1991; 109: 672-676.

23 Peral A, Dominguez-Godinez CO, Carracedo G, Pintor J. Therapeutic targets in dry eye syndrome. Drug News Perspect 2008; 21: 166-176.

24 Yeh S, Song XJ, Farley W, Li DQ, Stern ME, Pflugfelder SC. Apoptosis of ocular surface cells in experimentally induced dry eye. Invest Ophthalmol Vis Sci 2003; 44(1): 124-129.

25 Turner K, Pflugfelder SC, Ji Z, Feuer WJ, Stern M, Reis BL. Interleukin-6 levels in the conjunctival epithelium of patients with dry eye disease treated with cyclosporine ophthalmic emulsion. Cornea 2000; 19(4): 492-496.

26 Kunert KS, Tisdale AS, Stern ME, Smith JA, Gipson IK. Analysis of topical cyclosporine treatment of patients with dry eye syndrome: effect on conjunctival lymphocytes. Arch Ophthalmol 2000; 118(11): 1489-1496. 
27 Moore CP, McHugh JB, Thorne JG, Phillips TE. Effect of cyclosporine on conjunctival mucin in a canine keratoconjunctivitis sicca model. Invest Ophthalmol Vis Sci 2001; 42: 653-659.

28 Strong B, Farley W, Stern ME, Plugfelder SC. Topical cyclosporine inhibits conjunctival epithelial apoptosis in experimental murine keratoconjunctivitis sicca. Cornea 2005; 24: $80-85$.
29 Prummel MF, Mouritz MP, Berghout A, Krenning EP, van der Gaag R, Koornneef L et al. Prednisone and cyclosporine in the treatment of severe Graves' ophthalmopathy. N Engl J Med 1989; 321: 1353-1359.

30 Kahaly G, Schrezenmeir J, Krause U, Schwekert B, Meuer S, Muller W. Ciclosporin and prednisone v. prednisone in treatment of Graves' ophthalmopathy: a controlled, randomized and prospective study. Eur J Clin Invest 1986; 16: 415-422. 